\title{
Population pharmacokinetics of metoprolol in Chinese post-PCI patients
}

\author{
Hui Chen ${ }^{1}$, Huiwu Hong ${ }^{1}$, Wenjiao Zhao ${ }^{2}$, and Huan Wang ${ }^{1}$ \\ ${ }^{1}$ The shengli clinical Medical college of Fujian Medical University \\ ${ }^{2}$ Fuzhou second Hospital
}

September 11, 2020

\begin{abstract}
Abstract Aims: Metoprolol (MET) is widely used in post-PCI patient. This study aimed to establishment a new population pharmacokinetic (PPK) model of MET in Chinese post-PCI patients. Methods: Blood samples from subjects were collected and the CYP2D6*10 genotyping was detected by gene chip. The plasma concentration of MET was determined by LC-MS/MS. The PPK model of MET was developed by using NONMEM method. Pharmacogenetic analysis was carried out in the CYP2D6*10 genotyping. Results: Based on the data of 43 patients with coronary heart disease (CHD) after PCI, the final PPK model of MET was established as follows: ; A covariate analysis showed that only the CYP2D6 gene mutation had a significantly effect on the MET clearance rate. The CYP2D6*10 mutation could reduce the MET clearance rate. Compared with the CYP2D6*1*1 genotype the CYP2D6*1*10 genotype decreased by $36.6 \%$, the CYP2D*10/*10 decreased by $70.7 \%$ and showing a gene dose effect. Conclusions: The PPK model of MET established in this study is stable and reliable, which can provide reference for individual administration of MET. In Chinese patients with CHD after PCI, CYP2D6*10 gene mutation is one of the important factors affecting the MET clearance rate.
\end{abstract}

\section{Background:}

Metoprolol (MET) is the most commonly used selective $\beta 1$ receptor antagonist in the treatment of CHD. Almost all of MET is metabolized in the liver, and CYP2D6 mediates 70\%-80\% of MET metabolism ${ }^{[1]}$. In Chinese clinical practice, the dose of $\beta$-blockers in patients after PCI is seriously insufficient. In the results of China PEACE study, the daily dose of metoprolol tartrate tablets in AMI patients was [?]25mg ${ }^{[2]}$. This may be due to a variety of reasons, such as excessive consideration of the side effects of $\beta$-blockers, short hospitalization days, etc., but it may be related to the high CYP2D6*10 mutation rate in Chinese. CYP2D6 gene has significant genetic heterogeneity, of which nearly 100 mutants have been found so far ${ }^{[3]}$. Gene variation can affect the number and activity of coding enzymes. Several studies have confirmed that changes in pharmacokinetic parameters of MET are associated with CYP2D6 gene polymorphism ${ }^{[4-6]}$. However, there are obvious regional differences in CYP2D6 gene variation.

PPK is a new discipline in recent years, which is increasingly used in therapeutic drug monitoring (TDM) and clinical evaluation of new drug ${ }^{[7]}$. NONMEM software is not only the most widely used for PPK modeling, but also the most common and comprehensive software for feasibility evaluation. The establishment of drug PPK model by NONMEM method is approved by FDA ${ }^{[8]}$. At present, there are few studies on the pharmacokinetics of MET in China, in which the subjects are healthy volunteers. The clinical condition of patients post-PCI is uneven and there are many drugs used in combination, so it is difficult to estimate the appropriate dose of MET according to the pharmacokinetic parameters obtained in healthy people. And so far, there is no report on the application of NONMEM method to establish MET PPK model for Chinese patients with CHD. 
In our previous study, we found that the heart rate decreased more significantly in patients with homozygous mutation of CYP2D $6 * 10$ gene after treatment with MET than in patients with CYP2D $6 * 1$ allele ${ }^{[9]}$. Therefore, we wanted to further verify whether the variation of CYP2D6 gene affects the efficacy by changing the pharmacokinetics of MET. In this paper, a LC-MS/MS method was used to determine the serum concentration of MET, and the clinical data of patients were collected. NONMEM method was used to quantitatively investigate the effects of sex, age, CYP2D6 gene variation, liver and kidney function, combined medication and concomitant diseases on the pharmacokinetic parameters of MET. The PPK model of MET in Chinese patients with CHD after PCI was established to guide the individual administration of MET in these patients.

\section{Patients \& methods}

\subsection{Subjects}

A total of 43 post-PCI patients treated with MET were recruited, from February 2016 to February 2017 in the Department of Internal Medicine, Fujian Provincial Cardiovascular Disease Institute. Among them, $\beta$-blockers were given succinate sustained-release tablets (AstraZeneca, $47.5 \mathrm{mg} / \mathrm{d}$ ) for more than one month, and the heart rate was stable at 50-70 beats per minute, which was considered to have reached a stable state. All the subjects volunteered had signed the informed consent form before the trial and this study approved by the Ethics Committee of Fujian Provincial Hospital. The registration number of clinical trials in China is ChiCTR-TRC-13003596.

\subsection{Clinical data collection}

Those information from the subjects was collected 1): Demographic characteristics of patients: gender(SEX), age(AGE, weight(BW), height(HT), body mass index(BMI), etc. 2): Specific use of MET: starting date of taking MET, duration of taking medicine, dosage, blood concentration and specific time of collecting blood samples. 3): Complications: the concomitant diseases of more than $10 \%$ of the total population were included in the investigation factors, including hypertension and diabetes. 4): Combination of drugs: the combined drugs used by more than $10 \%$ of the total population were included in the study factors. Which including: aspirin (ASP), cholesterol (CHOL), ticagrelor (TIC), atorvastatin (ATO), rosuvastatin (ROS), ezetimibe (EZE), angiotensin converting enzyme inhibitors (ACEI), angiotensin receptor inhibitors (ARB), calcium channel blockers (CCB), trimetazidine (TRI), proton pump inhibitor (PPI), mucosta (MUC), etc. Blood biochemical conditions: liver function, renal function, blood lipids, electrolytes, etc. 5): Others: creatinine clearance $\left(\mathrm{C}_{\mathrm{Cr}}\right)$, left ventricular ejection fraction (LEF), number of coronary lesions (NCL), cardiac function classification (NYHA grade) and so on.

\subsection{Gene-chip technology to detect CYP2D6 genotype}

Genomic DNA was extracted from 250ul blood samples using a DNA Extraction Kit (Hunan Honghao Gene Biotechnology Co, Ltd, China). The PCR amplifications were carried out in a total volume of $25 \mu \mathrm{l}$ containing $5 \mu \mathrm{l}$ enzyme $19 \mu \mathrm{l}$ PCR reaction solution and $1 \mu$ l genomic DNA.PCR conditions included an initial denaturation at 37 for $10 \mathrm{~min}, 95$ for $5 \mathrm{~min}$ followed by 40 cycles of 95 for $40 \mathrm{~s}, 60$ for $40 \mathrm{~s}$, 72 for $40 \mathrm{~s}$ and then a final extension at 70 for $5 \mathrm{~min}$. Hybridization reaction includes $10 \mu \mathrm{l}$ PCR products, $8 \mu \mathrm{l}$ hybrid reaction liquid and $2 \mu \mathrm{l}$ positioning reference into the chip reaction zone in a constant $41 \mathrm{box}$ for $90 \mathrm{~min}$. Afterwards, remove the chip and put into three different cleaning mixtures for $1 \mathrm{~min}$. Finally, the dried slides were place in the GenePix4 100A (United States, MDC) scanner and used GenePix6.0 (United States, MDC) to quantitatively analyze the scan results. The analysis software performs genotype interpretation according to the set cut-off value.

\subsection{Analytical method of MET LC-MS/MS Assay}

Metoprolol tartrate (standards with $>99.9 \%$ purity), methanol, acetonitrile and Formic acid (all highperformance liquid chromatography (HPLC) grade) were get from the National Institutes for Food and Drug 
Control (Beijing, China). Liquid chromatography was performed on an ABsciex API3000 system (Applied Biosystem Co. Ltd., Waltham, USA), which was composed of electro spray ionization (ESI) Source, a Vacuum pump, an autosampler, and a column thermostat. The chromatographic column was AgilentEclipseXDBC18 $(4.6 \mathrm{~mm} \times 150 \mathrm{~mm}, 5 \mu \mathrm{m})$, the temperature was 30 , the mobile phase was methanol- $0.1 \%$ formic acid water, the gradient elution was used, and the injection volume was 10ul. Mass spectrometry conditions: electrospray ion source (electrospray ionization, ESI), positive ion mode, ion source temperature 500C, ion source spray voltage $5500 \mathrm{~V}$, ion pair quantitative analysis by multiple reaction monitoring (multiple reaction monitoring mode, MRM). Under the above conditions, MET mainly produces $[\mathrm{M}+\mathrm{H}]^{+}$ion front, which is $\mathrm{m} / \mathrm{z} 268.0$. Selective MS2 mass spectrum analysis showed that the main fragment ion of MET was m/z116.1.

The MET standard reference material of tartrate was prepared with acetonitrile to get 500mg/l MET standard reserve solution. And the 1.0mg/ l MET standard solution was prepared by step-by-step dilution of acetonitrile. Appropriate amount of $1.0 \mathrm{mg} / \mathrm{l} \mathrm{MET} \mathrm{standard} \mathrm{solution} \mathrm{was} \mathrm{removed} \mathrm{and} \mathrm{diluted} \mathrm{with}$ acetonitrile to prepare standard working solutions of different concentrations $(0.5 \mathrm{ng} / \mathrm{ml}, 1.0 \mathrm{ng} / \mathrm{ml}, 2.0 \mathrm{ng} / \mathrm{ml}$ $, 5.0 \mathrm{ng} / \mathrm{ml}, 10.0 \mathrm{ng} / \mathrm{ml}, 20.0 \mathrm{ng} / \mathrm{ml}, 40.0 \mathrm{ng} / \mathrm{ml}, 100.0 \mathrm{ng} / \mathrm{ml})$. The plasma samples were prepared by adding $400 \mu \mathrm{l}$ acetonitrile or corresponding standard working solution to the drug-containing plasma /blank plasma $400 \mathrm{\mu l}$ in the $1 \mathrm{ml}$ centrifuge tube. 2500rpm was swayed for $2 \mathrm{~min}$, then centrifuged with $10000 \mathrm{r} / \mathrm{min}$ at 10 for $5 \mathrm{~min}$ in a high-speed freezing centrifuge. The supernatant was absorbed through $0.45 \mathrm{um}$ organic phase filter membrane and analyzed by LC-MS/MS. In this experiment, the standard curve method was used to detect the plasma concentration of MET, using the concentration (X, ng/ml) as abscissa and the peak area of MET ion pair (Y) as ordinate to create the standard curve.

\section{Method Validation}

Accuracy and precision: The concentration of blank plasma samples with high, medium and low concentrations $(100 \mathrm{ng} / \mathrm{ml}, 40 \mathrm{ng} / \mathrm{ml}, 5 \mathrm{ng} / \mathrm{ml})$ was determined, and the percentage of the average value to the true value was used to measure the accuracy (method recovery). The plasma concentration of the above solution was determined at 5 different times in one day and 3 consecutive days. The intra-day RSD (relative standard deviation) was calculated as the standard of intra-day precision, the day-to-day RSD was calculated as the standard of day-to-day precision.

Matrix effect: The standard working solution was prepared with blank plasma and diluted with mobile phase respectively, and the standard working curve with the same concentration was prepared for determination. The ion response values of MET with the same concentration and different matrix were investigated, and the matrix effect (matrix effect, ME) of the sample solution was analyzed. The formula used is: $\mathrm{ME}=\mathrm{B} / \mathrm{A}$, where An and B represent the peak area of the target in the mobile phase and blank plasma matrix solution, respectively.

Stability: The stability of the drug in plasma was investigated by measuring the concentration changes of blank plasma samples with high, medium and low concentrations $(100 \mathrm{ng} / \mathrm{ml}, 40 \mathrm{ng} / \mathrm{ml}, 5 \mathrm{ng} / \mathrm{ml})$ at 4 for 7 days, -20 for 7 days, -20 for 30 days and repeated freezing and thawing at- 20 for 3 times(n=6).

\subsection{PPK model of MET by NONMEN method}

The one-compartment open model was used as the basic pharmacokinetic model, and the parameters of the model were calculated by NONMEM (Version 7.3, Icon Development Solutions, USA). The first-order conditional estimation (FOCE) with interaction method was selected throughout the model-building procedure to estimate the pharmacokinetic $(\mathrm{PK})$ parameters and their variability.

Using an exponential model to estimate individual variation: $C L_{i} / F\left(L h^{-1}\right)=\theta_{\mathrm{CL}} * e^{\eta_{i}} ; V_{i} / F(L)=\theta_{V} * e^{\eta_{i}}$. (In the formula, $\mathrm{C}_{\mathrm{Li}}$ :the clearance rate of MET in the i patient, unit was $\mathrm{l} / \mathrm{h}$;Vi:the apparent distribution volume of MET in patient I, unit was $\mathrm{L} ; \vartheta_{\mathrm{cL}}, \vartheta_{\mathrm{v}}$ : population typical values of CL and V; $\eta$ i: random effect factors among individuals; F:bioavailability). The selection of covariates follows the forward selection process and backward elimination process. The likelihood ratio was used to test the influence of each variable on the parameters. In the process of positive selection, the covariables that may have influence are substituted 
into the basic structure model respectively. After adding the covariable, the OFV is smaller than that of the infrastructure model OFV and the reduction value is more than 3.84. the covariable has a significant influence on the parameter estimation of the model, and the parameter is retained, otherwise it is eliminated. In the process of reverse elimination, one covariable was removed in the total regression model each time, and the OFV value changed after elimination. If the OFV value increased more than 6.64 , the covariable was retained in the final model.

The final model was validated based on statistical and graphical criteria. EXCEL was used to draw and the final model scatter plot to investigate the goodness of fit of the model, In the simplest model, scatter plot including: (i) observed (DV) vs. individual predicted concentrations (IPRED); (ii) DV vs. population predicted concentrations (PRED); (iii) weighted residuals (WRES) vs. time; (iv) WRES vs. PRED. And in the final model, scatter plot including: (i) WRES vs. ID; (ii) WRES vs. TIME, and (iii) WRES vs. PRED. Based on the original data, the stability and internal validity of the model are investigated by creating 2000 bootstrapped data sets and comparing with the original data by using the non-parametric Bootstrap method with resampling and replacement. Run NONMEM software "\$SIMULATION" and "\$ESTIMATION" module, apply Monte Carlo method to generate 2000 sets of simulation data from the final model to evaluate normalization prediction distribution error (NPDE), and verify the prediction performance and representativeness of the model.

\section{Results}

A total of 43 patients who met the criteria for enrollment were collected. The demographic and clinical characteristics of the patients are shown in the table 1 .

\subsection{Genotype analysis \& allelic frequencies}

The results of CYP2D $6 * 1 * 10$ detection by gene chip method are shown in the picture (Figure 1$)$. There were 8 patients with CYP2D6*1*1 genotype (18.61\%), 22 patients with CYP2D6*1*10 genotype (51.16\%) and 13 patients with CYP2D6*10*10 genotype (30.23\%). As shown in the Table 2 , the mutant allele frequency of $* 1$ is $44.19 \%$ and the $\mathrm{M}$ allele frequency of focus 10 is $55.81 \%$. The results of genetic analysis showed that Genotype distributions of CYP2D6 were in Hardy-Weinberg equilibrium ( $p>0.05$ )(Table 3). It is suggested that the selected patients come from a large and random mating group and are representative of the group.

\subsection{Determination of metoprolol in plasma by LC-MS/MS method}

\subsubsection{Specificity}

Under the above conditions of GC-MS, the MET mass spectrum is shown in figure 2. According to the ion abundance of the fragments, the qualitative and quantitative ion pairs of MET's are m/z268 ions m/z116.1. The chromatographic mass spectrum obtained by MET's LC-MS/MS is shown in figure 3. There is one more front in the patient's plasma chromatographic mass spectrum, but it is quite different from that of MET. The ion pair ratio is completely inconsistent with that of metoprolol ion pair, so it is determined to be a non-MET substance. From figure 3, it can be seen that endogenous substances, combined drugs and impurities do not interfere with the determination of MET.

\subsubsection{Standard curve and detection limit}

The regression equation of the standard curve of metoprolol plasma concentration established in this study is: $\mathrm{Y}=7.98^{*} 103^{*} \mathrm{X}+313(\mathrm{r}=1, \mathrm{n}=8)$ (figure 4). The linear range is $0.50-100 \mathrm{ng} / \mathrm{ml}$. The detection limit of metoprolol is based on ten times the standard working solution concentration of blank plasma with signalto-noise ratio. The detection limit of metoprolol is $0.33 \mathrm{ng} / \mathrm{ml}(\mathrm{S} / \mathrm{N}=10)$.

\subsubsection{Method recovery rate and precision}

The recovery rate, inter-day precision and intra-day precision of metoprolol method are shown in tables 4 and 5. Under three different concentrations, the deviation between the measured value and the true value is 
less than 5\%, and the RSD of intra-day and inter-day relative standard deviation is less than $5 \%$. It is proved that this method has high accuracy and good precision for the determination of metoprolol in plasma.

\subsubsection{Stability analysis}

The average concentration of MTE measured under different conditions is shown in Table 6, which shows that the sample has good stability under the conditions of preservation at 4,-20 and repeated freezing and thawing.

\subsection{Production of MTE PPK model}

\subsubsection{Production of the new model}

A one-compartment open model is used as the infrastructure model, and the simplest model result is obtained by NONME software. For the one-compartment model, there are only two parameters, CL and V. All the possible covariables were added to the minimalist model, and the effects on V and CL were investigated respectively. No covariates were found to affect V, but CYP, MUC, CHOL and EZE had effects on CL. The total regression model was established by the forward inclusion method, and the CYP, MUC and TC were retained. The backward elimination method is used to eliminate TC and MUC, to retain CYP covariates (Table 7). The final model formula of metoprolol is :

The estimated values of the parameters of the final model are shown in the Table 8 .

\subsubsection{Model evaluation}

From the scatter plot, it can be seen that the fitting degree of the final model PRED, IPRED and DV is better than that of the simplest model (Figure 5). The scatter diagrams of WRES vs.ID, TIME and PRED of the final model are shown in figure 6 . Most of the data points fall between \pm 2 , indicating that the final model fits well, and there is a good correlation between the predicted concentration and the measured concentration.

\subsubsection{Model Validation}

Verified by Bootstrap method, 2000 bootstrap databases were successfully fitted 1984 times, with a robustness rate of $99.2 \%$. The measured values are close to the estimated values of each parameter, and the absolute values of correlation deviations are less than $15 \%$. And the estimated values of each parameter fall within the $95 \% \mathrm{CI}$ of the estimated value of Bootstrap parameters (Table 9), indicating that the model is stable and effective.

\subsubsection{Model prediction ability}

The final model standardized prediction distribution are shown in Figure 7 .In that, the Wilcoxon signed Rank Sum Test $\mathrm{P}=0.486$ Fisher Variance Test $\mathrm{P}=0.983$ Shapiro-wick Wilk normal Distribution Test $\mathrm{P}=0.429$, and the total adjusted $\mathrm{P}$ value is 1 , suggesting that the model has good prediction ability and can be used to generate simulation data.

\subsubsection{The relationship between CYP2D6 genotype and MET dose}

We calculated the dose of MET in 43 patients. As shown in Table 10, only one patient with the genotype was CYP2D6*1*1 took MET 95mg; and one patient with CYP2D6*10*10 took MET $11.375 \mathrm{mg}$. The table shows that the variation of CYP2D6*10 needs to take a larger dose of MET to achieve clinical effect.

\section{Discussion}

The traditional pharmacokinetic study requires intensive and the whole process of blood collection, in order to accurately estimate the pharmacokinetic parameters ${ }^{[10]}$. This method is not feasible in clinical research, and the degree of matching of patients is not high, which limits its application. PPK is a method that combines traditional pharmacokinetic principles with statistical methods. Compared with traditional pharmacokinetics, PPK can deal with sparse and scattered data, which can be processed by $2-4$ blood samples. 
It can be used to quantitatively investigate the effects of related factors such as sex, age, gene variation, liver and kidney function, combined diseases, combined drugs and random effects on pharmacokinetics ${ }^{[11,12]}$. It can obtain pharmacokinetic models and parameters while understanding the pharmacokinetic characteristics of drugs. The drug administration information, drug metabolism in vivo and drug efficacy are combined to investigate the safety and effectiveness of drugs in a more comprehensive way ${ }^{[13,14]}$.

The final model showed that the CYP2D6*10 mutation had a smaller clearance rate than the wild type, which was consistent with the previous study found that the heart rate variability of CYP2D6*10 gene decreased more significantly ${ }^{[9]}$. Compared with wild type CYP2D6*10, the conversion of cytosine to thymine at position 188 on exon 1 caused proline to serine at position 34 of protease, resulting in the formation of low activity and unstable metabolic enzymes. The decrease of enzyme activity leads to the decrease of MET clearance rate $^{[15,16]}$. At the same dose of MET treatment, CYP2D6*10 mutation can improve the clinical efficacy of patients, and the side effects may also increase.

There are some studies have quantitatively investigated the effects of sex, age and CYP2D6 gene variation on the pharmacokinetic parameters of MET through the establishment of PPK model, but the results are not consistent. Eugene $\mathrm{A}^{[17]}$ published articles on gender-based differences in MET clearance in elderly patients with multiple complications. The results showed that the clearance rate of female elderly patients decreased by $57 \%$ compared with men with similar physical conditions. In addition, in another article that for healthy young people, the MET clearance rate of women was about $50 \%$ lower than that of men ${ }^{[18]}$. On the other hand, Taguchi $\mathrm{M}^{[19]}$ reported that under the routine treatment dose of MET in Japanese, sex did not affect the clearance rate of MET, while age and CYP2D6*10 gene mutation affected the clearance rate, which showed that the clearance rate of elderly patients ( $>70$ years old) was $26 \%$ lower than that of young patients ( $<70$ years old). The CYP2D6*10 homozygous mutation was $64 \%$ lower than that of the CYP2D6* $1 /{ }^{*} 1$ genotype. The results of our study showed that the variation of CYP2D6*10 had a significant effect on MET clearance, which showed that the clearance rate of CYP2D $6 * 1 / * 10$ genotype decreased by $36.6 \%$ compared with that of CYP2D6*1/*1 genotype, which was similar to that of Taguchi $\mathrm{M}^{[19]}$. But this paper did not find that age and sex can affected the clearance rate of MET.

In this study, the PPK model of MET in Chinese patients with CHD after PCI was established for the first time by NONMEM software. Our results are consistent with clinical practice, the clearance rate of CYP2D6*10/*10 is significantly reduced, oral lower dose can achieve clinical efficacy, that can be used to guide the individual use of MET. In practice, when use MET to treat CHD, we choose to start with a small dose and titrate gradually to the target dose, thus prolonging the hospitalization time of patients. Actually, the turnover rate of patients after PCI is fast, and most patients do not reach the target dose when they are discharged from hospital, so they can't achieve the best therapeutic effect. All in all, CYP2D6 genotyping can be used to estimate the initial dose of MET, which provides a reference for individualized treatment of MET.

There are some limitations in our study. Due to the limited number of subjects, the PPK model of MET was established and internally validated only. External validation will be carried out after applying it in clinical practice.

\section{Conclusions}

The PPK model of MET established in this study is stable and reliable, which can provide reference for individual administration of MET. CYP2D6*10 gene mutation is one of the important factors affecting the clearance rate of MET. According to the results of this study, the initial dose of MET for post-PCI patients can be estimated by measuring CYP2D6 genotype, and titrated to the target dose as soon as possible, so as to shorten the hospitalization time and greatly improve the rate of MET dose reaching the standard. 


\section{:::::::}
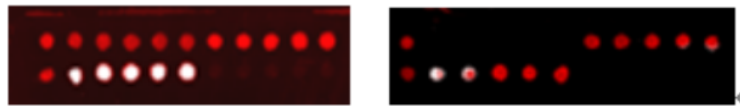

Figure 1: The result of CYP2D6 detection by gene chip method
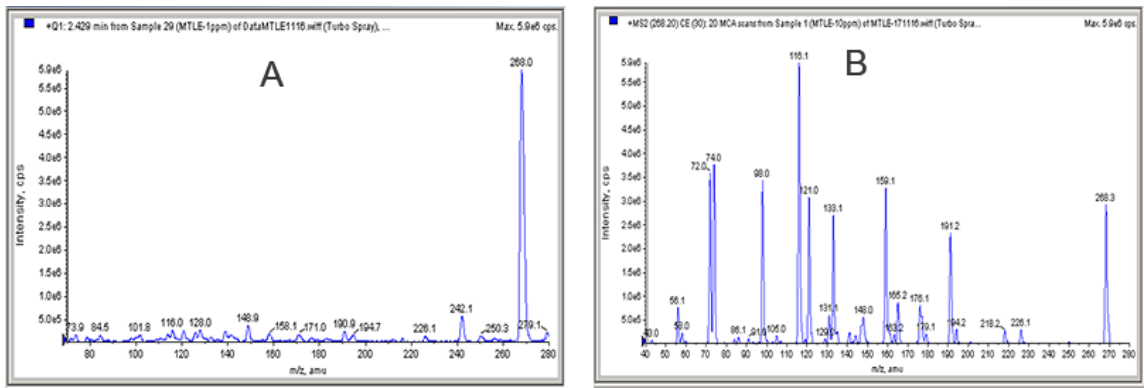

Figure 2: MET mass spectrogram; (A)The MET primary mass spectrogram; (B)The MET secondary mass spectrogram

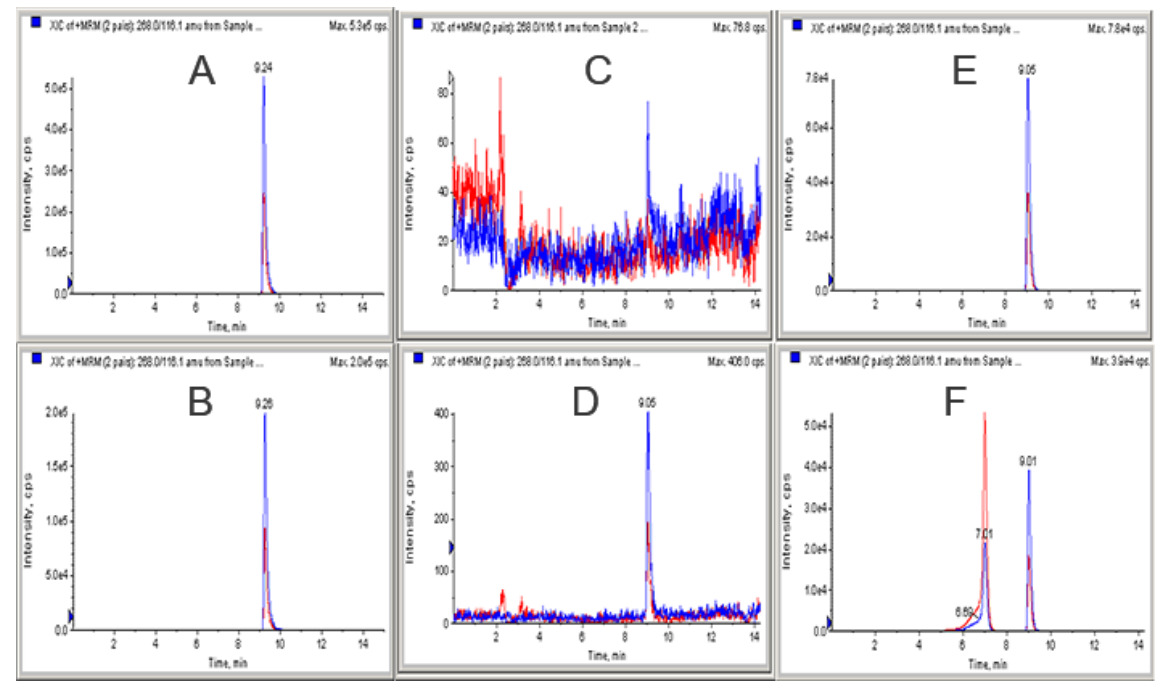

Figure 3: MET chromatographic mass spectrogram determined by LC-MS/MS. (A) Tartrate MET; (B) Succinate MET sustained release tablets; (C) Blank plasma; (D) Blank plasma + standard sample (0.05ng/mL); (E) Blank plasma + standard sample (100ng/mL); (F) Patient plasma. 


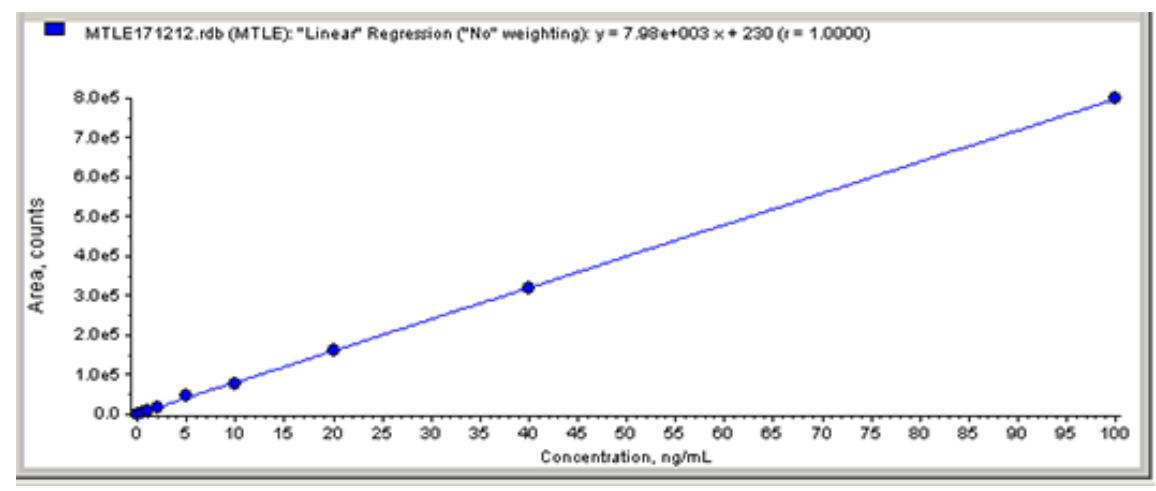

Figure 4: Standard curve for determination of metoprolol in plasma
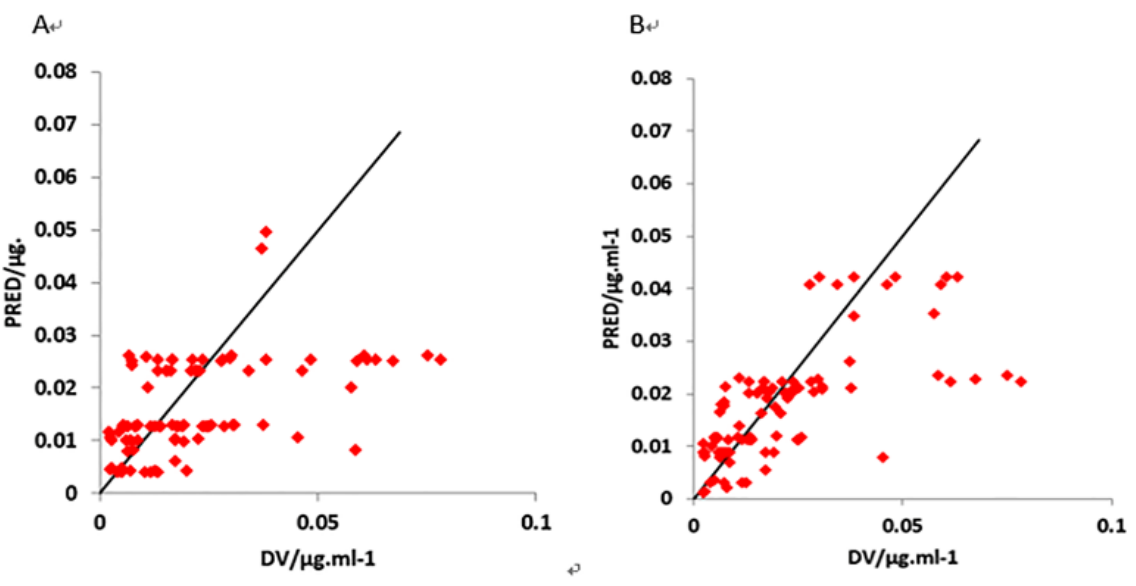

$$
\text { C }
$$

D
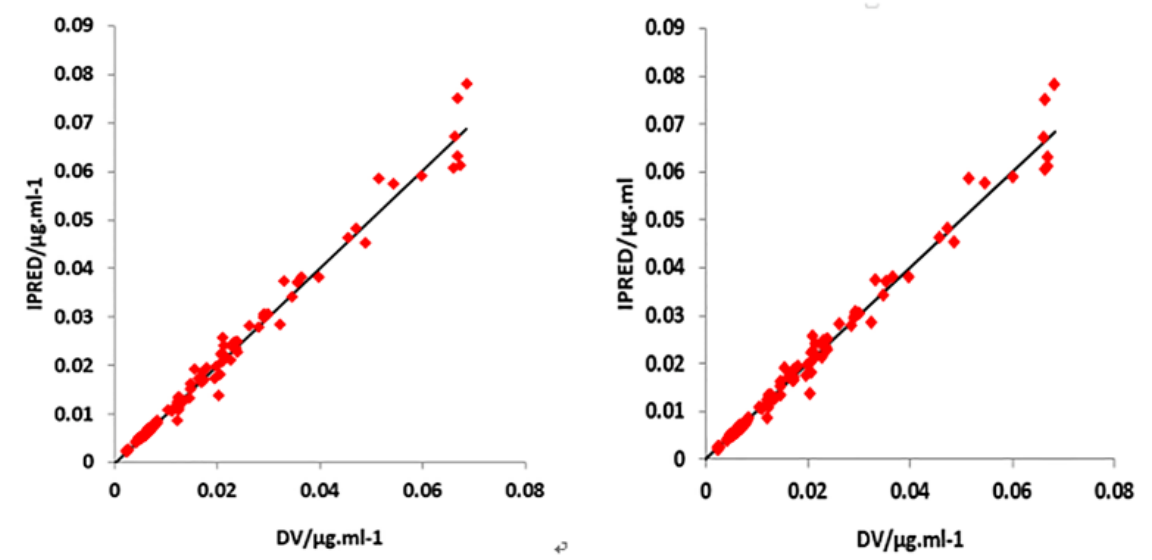

Figure 5: Scatter plot of predicted and measured values.(A) The scatter plot of the simplest model group predicted value and measured value; (B)The final model group predicted value and measured value scatter chart; (C)The simplest model individual predicted value and measured value scatter plot; (D)The final model individual predicted value and measured value scatter chart 
A

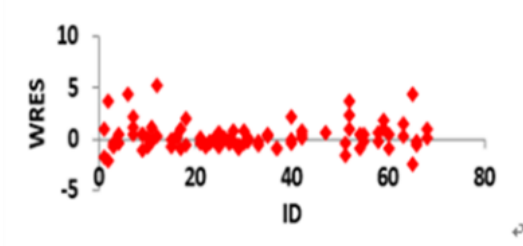

C
B

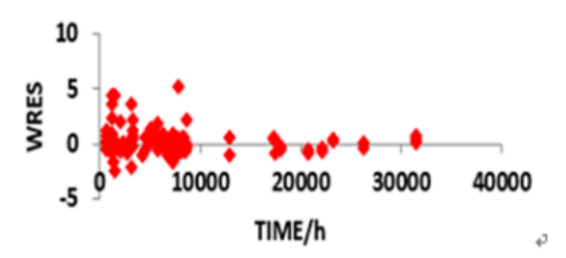

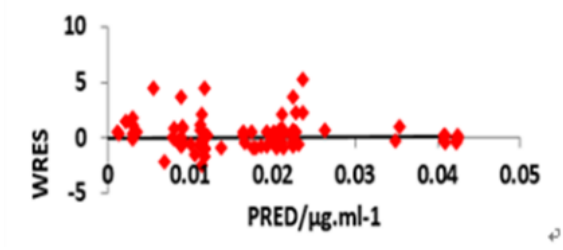

Figure 6: Final model weighted residual scatter diagram. (A)Weighted residual and patient number scatter plot; (B)Weighted residual and time scatter plot; (C)Weighted residual and group predictive scatter plot; where WRES=DV-PRED/PF.
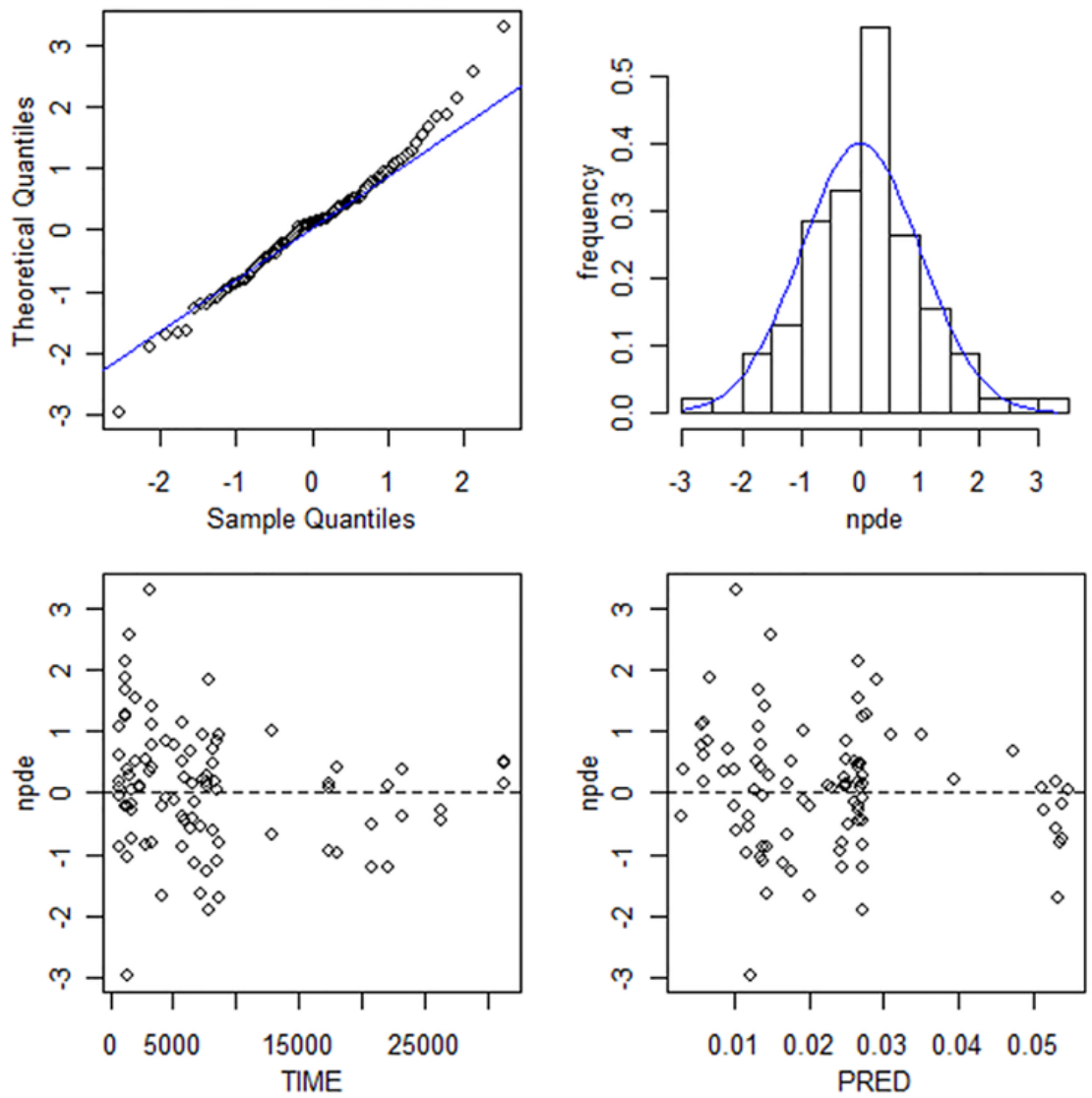

Figure 7: Normalized prediction distribution errors (NPDE) analysis for the MET final model. NPDE: QQ-plot of the distribution of the NPDE versus the theoretical N $(0,1)$ distribution (upper left). Histogram 
of the distribution of the NPDE, with the density of the standard Gaussian distribution overlaid (upper right); NPDE and TIME scatter plot (lower left); NPDE and PRED scatter plot (lower right).

Table 1: Population data characteristics of 43 patients

\begin{tabular}{llll}
\hline Measurement date & Measurement date & Categorical date & Categorical date \\
AGE & $63(47-75)$ & SEX(man/female) & $32 / 11$ \\
BW $(\mathrm{kg})$ & $67.09(50-98)$ & NYHA I/II & $18 / 25$ \\
HT(cm) & $166.21(151-184)$ & CYP2D6 1/2/3 & $8 / 22 / 3$ \\
BMI $\left(\mathrm{kg} . \mathrm{m}^{-2}\right)$ & $24.23(17.72-31.6)$ & ASP & $36(83.72 \%)$ \\
ALT $(\mathrm{U} / \mathrm{l})$ & $26.51(10-108)$ & CLO & $34(79.07 \%)$ \\
AST $(\mathrm{U} / \mathrm{l})$ & $23.95(10-62)$ & TIC & $6(13.95 \%)$ \\
TG $(\mathrm{mmol} / \mathrm{l})$ & $1.48(0.47-4.05)$ & ATO & $21(48.84 \%)$ \\
TC $(\mathrm{mmol} / \mathrm{l})$ & $3.79(1.98-5.73)$ & ROS & $17(39.53 \%)$ \\
HDL $(\mathrm{mmol} / \mathrm{l})$ & $1.15(0.62-2.35)$ & EZE & $6(13.95 \%)$ \\
$\mathrm{LDL}(\mathrm{mmol} / \mathrm{l})$ & $2.41(0.58-4.11)$ & ACEI/ARB & $31(72.09 \%)$ \\
$\mathrm{BUN}(\mathrm{mmol} / \mathrm{l})$ & $5.13(3.2-9.3)$ & CCB & $12(27.91 \%)$ \\
$\mathrm{CR}(\mathrm{umol} / \mathrm{l})$ & $75.23(42-104)$ & TRI & $19(44.19 \%)$ \\
$\mathrm{Ccr}(\mathrm{ml} / \mathrm{min})$ & $83.18(52.67-149.3)$ & PPI & $21(48.84 \%)$ \\
$\mathrm{K}(\mathrm{mmol} / \mathrm{l})$ & $4.21(3.6-5.0)$ & MUC & $5(10.63 \%)$ \\
NA $(\mathrm{mmol} / \mathrm{l})$ & $141.09(137-146)$ & HBP & $33(76.74 \%)$ \\
$\mathrm{LEF}(\%)$ & $59.53(41-69)$ & DM & $10(23.26 \%)$ \\
\hline
\end{tabular}

BMI : Body mass index;ALT : Glutamic pyruvic transaminase; AST : Aspartate transaminase; TG : Triglyceride; TC : Serum total cholesterol; HDL : High density lipoprotein; LDL : Low density lipoprotein; BUN : Blood urea nitrogen; CR : Creatinine; Ccr : Creatinine clearance rate; LVEF : Left ventricular ejection fraction; NYHA : The NYHA function classification; ASP : Aspirin; CHOL: Cholesterol;TIC : Ticagrelor; ATO : Atorvastatin calcium;ROS : Rosuvastatin calcium; EZE : Ezetimibe;ACEI/ARB : Angiotensin converting enzyme inhibitor / Angiotensin receptor inhibitor; CCB : Calcium channel blocker; TRI : Trimetazidine; PPI : Proton pump inhibitor; MUC : Mucosta; HBP : Hypertension; DM : Diabetes Mellitus.

Table 2: CYP2D6 genotype distribution of 43 patients

\begin{tabular}{|c|c|c|c|c|c|c|}
\hline & & & Genotype frequency & Genotype frequency & Genotype frequency & $\mathrm{M}$ allele frequency \\
\hline CYP2D6 & CYP2D6 & $* 1 * 1$ & $* 1 * 1$ & $* 1 * 10$ & $* 10 * 10$ & $* 1$ \\
\hline Patients & $8(18.61)$ & $8(18.61)$ & $8(18.61)$ & $22(51.16)$ & $3(30.23)$ & 44.19 \\
\hline
\end{tabular}

$* 1 \mathrm{M}$ allele frequency $=(\mathrm{CYP} 2 \mathrm{D} 6 * 1 * 1 \times 2+\mathrm{CYP} 2 \mathrm{D} 6 * 1 * 10) / 43 \times 2$

$* 10 \mathrm{M}$ allele frequency $=(\mathrm{CYP} 2 \mathrm{D} 6 * 10 * 10 \times 2+\mathrm{CYP} 2 \mathrm{D} 6 * 1 * 10) / 43 \times 2$

Table 3: Hardy-Weinberg balance analysis table

\begin{tabular}{lllllll}
\hline & $* 1 * 1(\mathrm{n}, \%)$ & $* 1 * 1(\mathrm{n}, \%)$ & $* 1 * 10(\mathrm{n}, \%)$ & $* 10 * 10(\mathrm{n}, \%)$ & $\mathrm{X}^{2}$ & $\mathrm{P}$ \\
\hline Observed value & Observed value & $8(18.61)$ & $22(51.16)$ & $13(30.23)$ & 0.059 & \multirow{2}{*}{0.05} \\
Theoretical frequency & Theoretical frequency & 19.53 & 49.32 & 31.15 & & \\
Theoretical value & Theoretical value & 8.4 & 21.21 & 13.39 & & \\
\hline
\end{tabular}


Table 4: Recovery rate test of the $\operatorname{method}(\mathrm{n}=6)$

\begin{tabular}{lll}
\hline Con $(\mathrm{ng} / \mathrm{ml})$ & $? \mathrm{X}(\mathrm{ng} / \mathrm{ml})$ & Recovery rate $(\%)$ \\
\hline 5 & 4.86 & 97.2 \\
40 & 40.56 & 101.4 \\
100 & 100.58 & 100.06 \\
\hline
\end{tabular}

Table 5: Metoprolol intra-day \& daytime precision test $(\mathrm{n}=6)$

\begin{tabular}{lllllll}
\hline Con $(\mathrm{ng} / \mathrm{ml})$ & Intra-day & Intra-day & Intra-day & Daytime & Daytime & Daytime \\
\hline & $? \mathrm{X}(\mathrm{ng} / \mathrm{ml})$ & $\mathrm{SD}$ & $\mathrm{RSD}(\%)$ & $? \mathrm{X}(\mathrm{ng} / \mathrm{ml})$ & $\mathrm{SD}$ & $\mathrm{RSD}(\%)$ \\
5 & 4.86 & 0.23 & 4.73 & 4.92 & 0.18 & 3.66 \\
40 & 40.23 & 1.03 & 2.56 & 40.36 & 1.37 & 3.39 \\
100 & 101.35 & 1.36 & 1.34 & 100.89 & 1.01 & 1 \\
\hline
\end{tabular}

Table 6: The average concentration of MTE under different conditions(n=6)

\begin{tabular}{lllll}
\hline Con $(\mathrm{ng} / \mathrm{ml})$ & $4 \mathrm{~d}_{\mathbf{7}}$ & $-20 \mathrm{~d}_{\mathbf{7}}$ & $-20 \mathrm{~d}_{\mathbf{3 0}}$ & Freezing and thawing \\
\hline 5 & 4.89 & 4.86 & 4.89 & 4.79 \\
40 & 41.32 & 40.97 & 40.97 & 40.69 \\
100 & 100.76 & 100.08 & 101.22 & 99.79 \\
\hline
\end{tabular}

Table 7:The process of establishing the final model

\begin{tabular}{llllll}
\hline Model & Add covariate & Remove covariates & OFV & ${ }^{O} F V$ & Remarks \\
\hline Base model & & & -840.36 & - & \\
& CYP & & -853.96 & $-13.604^{\mathrm{a}}$ & \\
& MUC & & -845.08 & $-4.719^{\mathrm{a}}$ & \\
& CHOLL & & -844.43 & $-4.067^{\mathrm{a}}$ & \\
& EZE & & -844.27 & $-3.91^{\mathrm{a}}$ & \\
& CYP & & -853.96 & $-13.604^{\mathrm{b}}$ & Keep \\
& MUC & & -859.5 & $-5.536^{\mathrm{b}}$ & Keep \\
& CHOL & & -865.72 & $-6.22^{\mathrm{b}}$ & Keep \\
Full regression model & CYE & -867.72 & $0^{\mathrm{b}}$ & Remove \\
Backward elimination & CYP+MUC+CHOL & & -865.72 & - & \\
& CYP & CHOL & -859.5 & $6.22^{\mathrm{b}}$ & Remove \\
& - & MUC & -853.96 & $5.536^{\mathrm{b}}$ & Remove \\
Final model & CYP & CYP & -844.43 & $9.537^{\mathrm{b}}$ & Keep \\
\hline
\end{tabular}

CYP: CYP2D6 genotype; MUC : Mucosta; EZE : Ezetimibe; CHOL: Cholesterol

a: Objective function value - Objective function value of the simplest model;

b : Objective function value - Previous objective function value 
Table 8:The process of establishing the final model

\begin{tabular}{|c|c|c|}
\hline Parameters & Estimated value & $\operatorname{RSE}(\%)$ \\
\hline$\vartheta \vartheta_{\Lambda}$ & 201 & 21.4 \\
\hline$\vartheta$ & 1220 & 9.8 \\
\hline$\vartheta “ \Psi \Pi 2$ & 0.634 & 29.3 \\
\hline$\vartheta " \Psi \Pi 3$ & 0.283 & 24.5 \\
\hline$\omega * \Lambda$ & 0.741 & 14.1 \\
\hline$\omega$ & 0.306 & 21.3 \\
\hline$\sigma$ & 0.125 & 14.1 \\
\hline
\end{tabular}

$\vartheta^{\prime} \Lambda$ : Typical population value of MET clearance rate; $\boldsymbol{\vartheta}^{\circ}:$ Typical value of MET apparent distribution

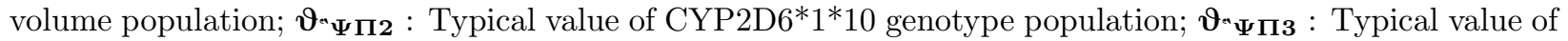
CYP2D6*10*10 genotype population; $\boldsymbol{\omega}^{\text {" } \boldsymbol{\Lambda}}$ : The inter-individual variation of CL; $\boldsymbol{\omega}^{\boldsymbol{*}}$ : The inter-individual variation of $\mathrm{V} ; \sigma$ : Intra-individual variation.

Table 9: Final model Bootstrap analysis results

\begin{tabular}{|c|c|c|c|c|}
\hline Parameter & Value & Bootstrap & Bootstrap & relative bias(\%) \\
\hline & & Median & $95 \% \mathrm{CI}$ & \\
\hline$\vartheta^{\prime} \Lambda$ & 201 & 193 & $134-319$ & -3.98 \\
\hline$\vartheta$ & 1220 & 1270 & $1030-2050$ & 4.09 \\
\hline$\vartheta$ & 0.634 & 0.659 & $0.356-1.1$ & 3.94 \\
\hline$\vartheta^{\prime \prime} \Psi \Pi 3$ & 0.293 & 0.291 & $0.167-0.457$ & -0.68 \\
\hline$\omega^{n} \Lambda$ & 0.741 & 0.677 & $0.479-0.895$ & -8.63 \\
\hline$\omega$ & 0.306 & 0.305 & $0.005-0.709$ & -0.33 \\
\hline$\sigma$ & 0.125 & 0.122 & 0.0796-.169 & -2.40 \\
\hline
\end{tabular}

$\vartheta_{{ }^{\prime} \Lambda}$ : Typical population value of MET clearance rate; $\vartheta^{\circ}:$ Typical value of MET apparent distribution volume population; $\boldsymbol{\vartheta}^{\prime} \Psi \Pi \mathbf{2}$ : Typical value of CYP2D6*1*10 genotype population; $\boldsymbol{\vartheta}^{\wedge}{ }^{*} \Psi \Pi \mathbf{3}$ : Typical value of CYP2D $6 * 10 * 10$ genotype population; $\boldsymbol{\omega}^{*} \boldsymbol{\Lambda}$ : The inter-individual variation of $\mathrm{CL} ; \boldsymbol{\omega}^{*}$ : The inter-individual variation of $\mathrm{V} ; \sigma$ : Intra-individual variation.

Table 10: CYP2D6 genotype and MET dose

\begin{tabular}{|c|c|c|c|c|}
\hline & $11.375(\mathrm{mg})$ & $23.75(\mathrm{mg})$ & $47.5(\mathrm{mg})$ & $95(\mathrm{mg})$ \\
\hline $1 * 1(\mathrm{n} / \%)$ & $0(0.00)$ & $4(50.00)$ & $3(37.50)$ & $1(12.50)$ \\
\hline $1 * 10(\mathrm{n} / \%)$ & $0(0.00)$ & $10(54.55)$ & $10(45.45)$ & $0(0.00)$ \\
\hline $10 * 10(\mathrm{n} / \%)$ & $1(7.69)$ & $7(53.85)$ & $5(38.46)$ & $0(0.00)$ \\
\hline
\end{tabular}

\section{References:}

1. Kaila N, Straka RJ, Brundage RC. Mixture models and subpopulation classification: a pharmacokinetic simulation study and application to metoprolol CYP2D6 phenotype. J Pharmacokinet Pharmacodyn 2007; 34: $141-56$.

2. Jing, Li, Xi, et al. ST-segment elevation myocardial infarction in China from 2001 to 2011 (the China PEACE-Retrospective Acute Myocardial Infarction Study): a retrospective analysis of hospital data. 2015.

3. Frank D, Jaehde U, Fuhr U. Evaluation of probe drugs and pharmacokinetic metrics for CYP2D6 pheno- 
typing. Eur J Clin Pharmacol 2007; 63: 321-33.

4. D L, W D, Y C, et al. Effect of pathway training on rest heart rate and the application of $\beta$-blocker in coronary heart disease patients: an open-label, multi-center, prospective study. Zhonghua yi xue za zhi 2015; 95: 2272-6.

5. Fox K, Ford I, Steg PG, et al. Relationship between ivabradine treatment and cardiovascular outcomes in patients with stable coronary artery disease and left ventricular systolic dysfunction with limiting angina: a subgroup analysis of the randomized, controlled BEAUTIFUL trial. Eur Heart J 2009; 30: 2337-45.

6. Ibanez B, James S, Agewall S, et al. 2017 ESC Guidelines for the management of acute myocardial infarction in patients presenting with ST-segment elevation. Rev Esp Cardiol (Engl Ed) 2017; 70: 1082.

7. Aarons L. Population pharmacokinetics: theory and practice. Br J Clin Pharmacol 1991; 32: 669-70.

8. FAD. Guidance for industry on Population Pharmacokinetics; availability. . 1999; 64: 6663-4.

9. Gao X, Wang H, Chen HJP. Impact of CYP2D6 and ADRB1 polymorphisms on heart rate of post-PCI patients treated with metoprolol. 2017.

10. Duan JJJocp, therapeutics. Applications of population pharmacokinetics in current drug labelling. 2007; 32: $57-79$.

11. Chan Kwong A, Calvier E, Fabre D, et al. Prior information for population pharmacokinetic and pharmacokinetic/pharmacodynamic analysis: overview and guidance with a focus on the NONMEM PRIOR subroutine. 2020.

12. Aljutayli A, Marsot A, Nekka FJCp. An Update on Population Pharmacokinetic Analyses of Vancomycin, Part I: In Adults. 2020; 59: 671-98.

13. Abdel Jalil M, Abdullah N, Alsous M, et al. A systematic review of population pharmacokinetic analyses of digoxin in the paediatric population. 2020; 86: 1267-80.

14. Sassen S, Zwaan C, van der Sluis I, et al. Pharmacokinetics and population pharmacokinetics in pediatric oncology. 2020; 67: e28132.

15. Bagheri A, Kamalidehghan B, Haghshenas M, et al. Prevalence of the CYP2D6*10 (C100T), *4 (G1846A), and *14 (G1758A) alleles among Iranians of different ethnicities. Drug Des Devel Ther 2015; 9: $2627-34$.

16. Nozawa T, Taguchi M, Tahara K, et al. Influence of CYP2D6 genotype on metoprolol plasma concentration and beta-adrenergic inhibition during long-term treatment: a comparison with bisoprolol. J Cardiovasc Pharmacol 2005; 46: 713-20.

17. Eugene AR. Metoprolol Dose Equivalence in Adult Men and Women Based on Gender Differences: Pharmacokinetic Modeling and Simulations. Med Sci (Basel) 2016; 4.

18. Eugene AR. Gender based Dosing of Metoprolol in the Elderly using Population Pharmacokinetic Modeling and Simulations. Int J Clin Pharmacol Toxicol 2016; 5: 209-15.

19. Taguchi M, Nozawa T, Mizumaki K, et al. Nonlinear mixed effects model analysis of the pharmacokinetics of metoprolol in routinely treated Japanese patients. Biol Pharm Bull 2004; 27: 1642-8.

$* 1 * 1$

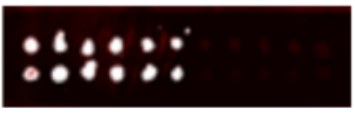

$* 1 * 10$

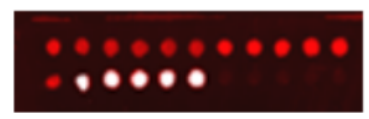

$* 10 * 10$

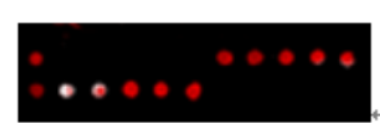



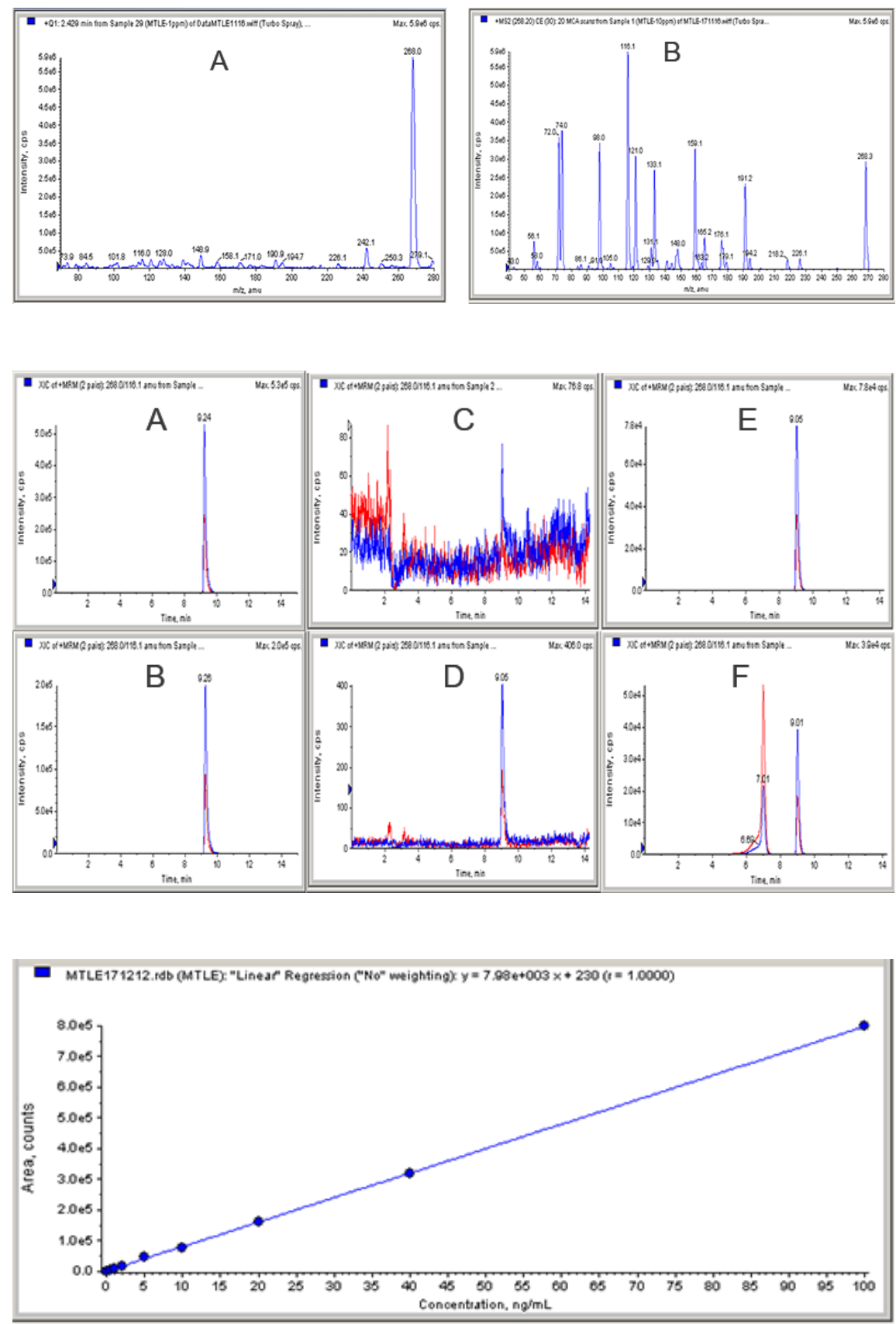

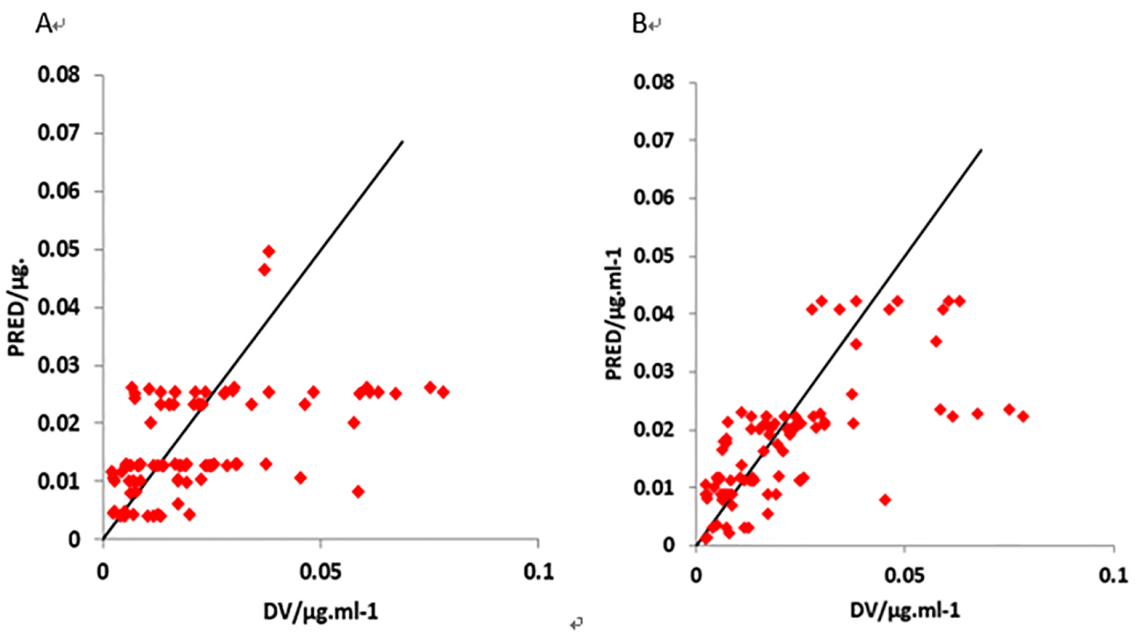

C

D
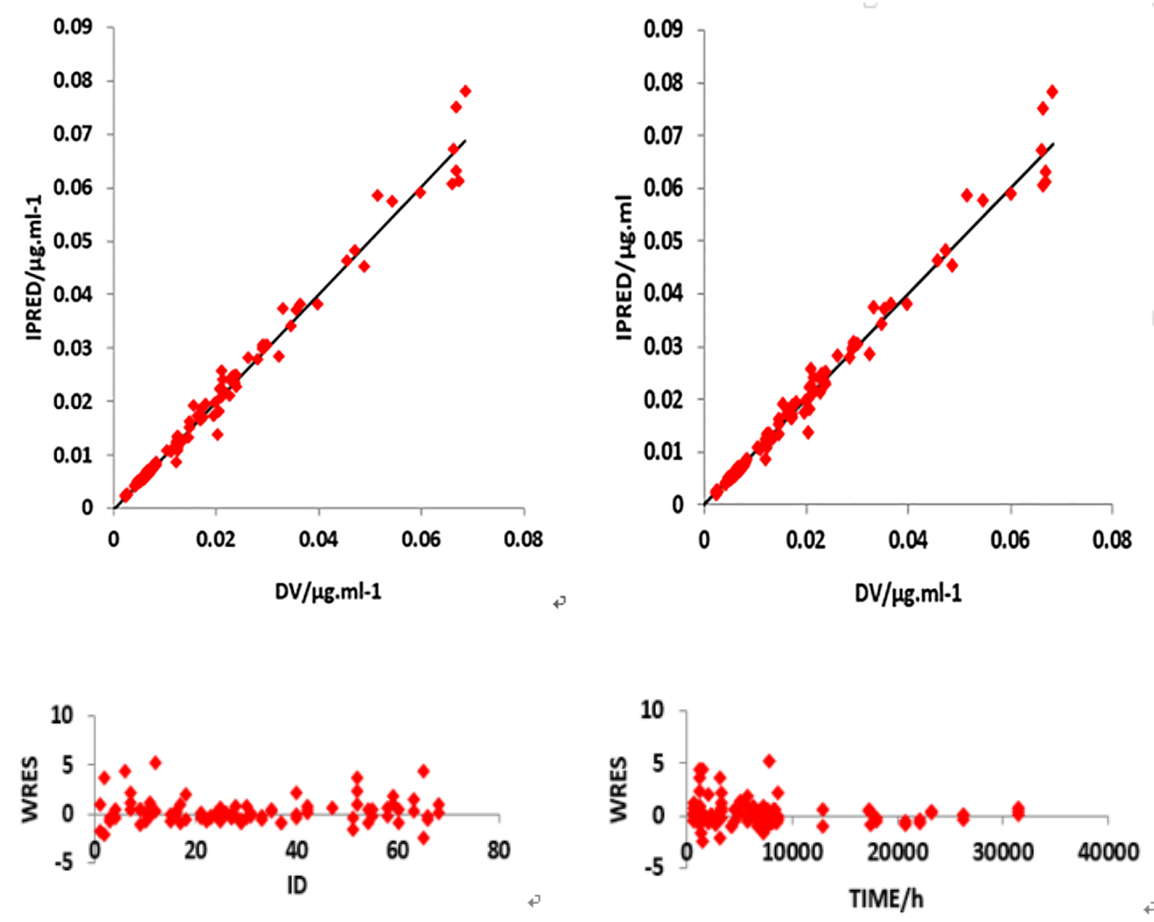

C

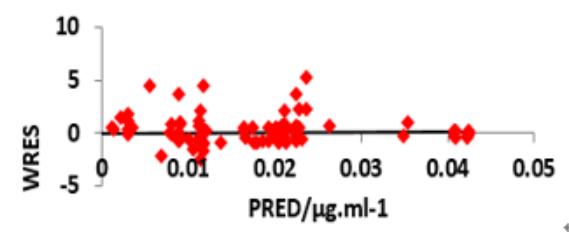



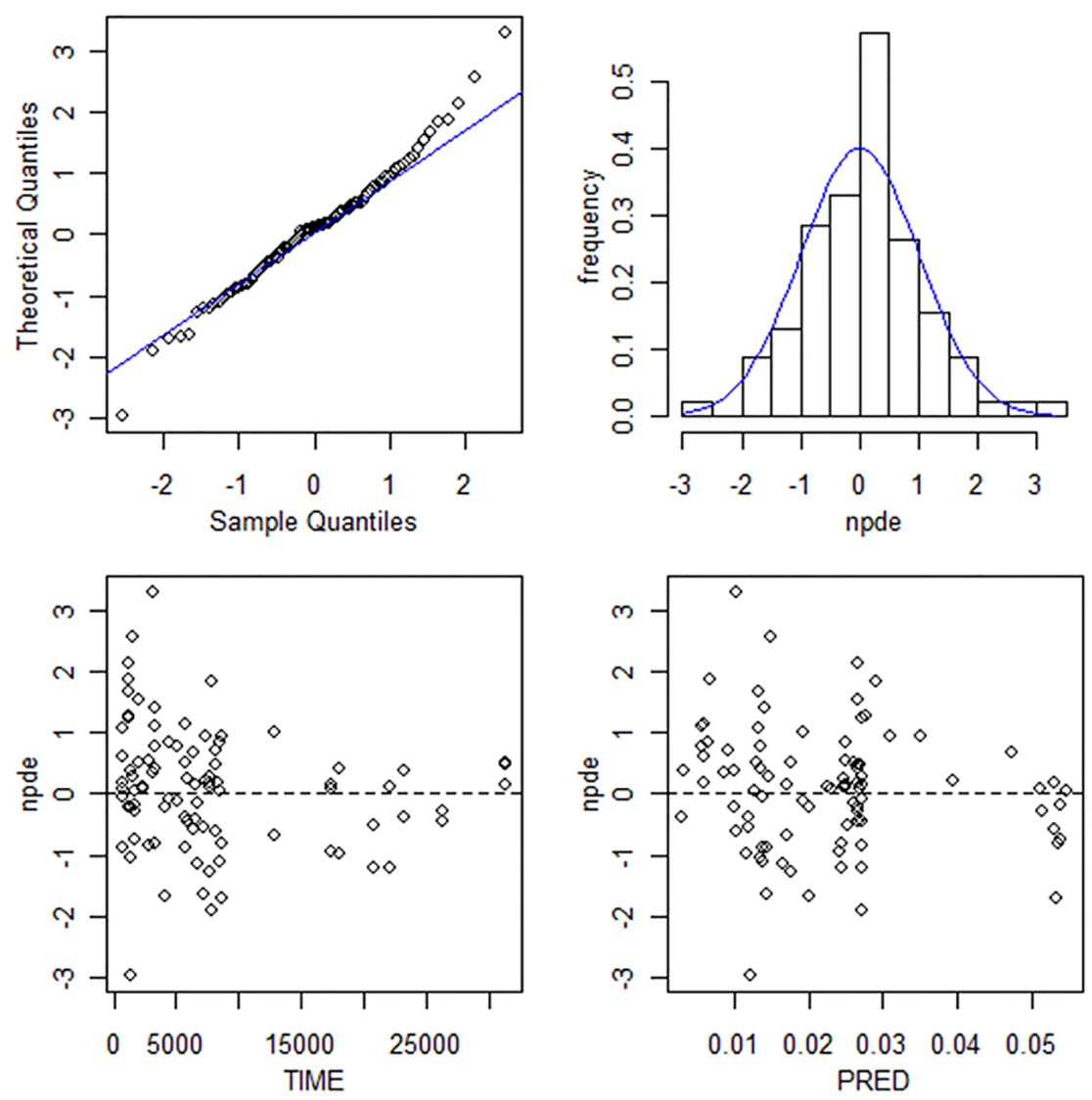

\section{Hosted file}

Table-1.docx available at https://authorea.com/users/357357/articles/479920-populationpharmacokinetics-of-metoprolol-in-chinese-post-pci-patients

\section{Hosted file}

Table-2.docx available at https://authorea.com/users/357357/articles/479920-populationpharmacokinetics-of-metoprolol-in-chinese-post-pci-patients

\section{Hosted file}

Table-3.docx available at https://authorea.com/users/357357/articles/479920-populationpharmacokinetics-of-metoprolol-in-chinese-post-pci-patients

\section{Hosted file}

Table-4.docx available at https://authorea.com/users/357357/articles/479920-populationpharmacokinetics-of-metoprolol-in-chinese-post-pci-patients

\section{Hosted file}

Table-5.docx available at https://authorea.com/users/357357/articles/479920-populationpharmacokinetics-of-metoprolol-in-chinese-post-pci-patients

\section{Hosted file}


Table-6.docx available at https://authorea.com/users/357357/articles/479920-populationpharmacokinetics-of-metoprolol-in-chinese-post-pci-patients

\section{Hosted file}

Table-7.docx available at https://authorea.com/users/357357/articles/479920-populationpharmacokinetics-of-metoprolol-in-chinese-post-pci-patients

\section{Hosted file}

Table-8.docx available at https://authorea.com/users/357357/articles/479920-populationpharmacokinetics-of-metoprolol-in-chinese-post-pci-patients

\section{Hosted file}

Table-9.docx available at https://authorea.com/users/357357/articles/479920-populationpharmacokinetics-of-metoprolol-in-chinese-post-pci-patients

\section{Hosted file}

Table-10.docx available at https://authorea.com/users/357357/articles/479920-populationpharmacokinetics-of-metoprolol-in-chinese-post-pci-patients 